\title{
Valor preditivo de marcadores séricos de fibrose hepática em pacientes portadores de hepatite crônica viral C
}

\section{Predictive value of serum markers of hepatic fibrosis in patients with chronic hepatitis $C$}

Leila Maria Soares Tojal de Barros Lima'; João Roberto Maciel Martins²; Helena Bonclani Nader³; Celina Maria Costa Lacet ${ }^{4}$; Maria do Carmo Lins Vasconcelos Balwani ${ }^{5}$; Maria Aparecida da Silva Pinhal ${ }^{6}$

\begin{abstract}
unitermos
Marcadores séricos de

fibrose hepática

Ácido hialurônico

Fibrose hepática

Hepatite crônica C

\section{resumo}

Introdução: Os marcadores séricos têm sido empregados na avaliação da fibrose hepática em pacientes portadores de hepatite crônica C (HCC). Objetivos: Avaliar a capacidade do índice aspartato aminotransferase (AST)/alanina aminotransferase (ALT), dos níveis séricos de gama-glutamiltransferase (GGT), contagem de plaquetas, do índice AST/plaquetas (APRI) e do ácido hialurônico (AH) em predizer a intensidade da fibrose hepática na HCC e a variação desses marcadores após tratamento com interferon. Pacientes e métodos: $\mathrm{Em} 72$ pacientes portadores de hepatite $\mathrm{C}$ determinamos no soro $\mathrm{o}$ índice AST/ALT, GGT, plaquetas, índice APRI (obtido pelo quociente AST/plaquetas) e o AH, que foram comparados ao estadiamento histológico, segundo os critérios de METAVIR. Receberam tratamento com interferon e ribavirina 65 pacientes. Os indivíduos que concluíram o tratamento $(n=33)$ realizaram nova dosagem dos marcadores séricos de fibrose para comparar com os níveis pré-tratamento. Resultados: Observamos que a GGT, a contagem de plaquetas, o índice APRI e o AH se correlacionaram com estádio de doença hepática $(p<0,01)$, exceto o índice AST/ALT. A análise das áreas sob as curvas ROC (AUC) evidenciaram que a melhor associação com estadiamento hepático foi para o índice APRI e a dosagem sérica do AH: AUC (APRI) = 0,85 e AUC $(A H)=0,86$. Na avaliação pós-terapia com interferon, apenas a GGT e o índice APRI apresentaram redução de seus níveis $(p<0,05)$. Conclusão: $\mathrm{O} A H$ e o índice APRI apresentaram maior acurácia no estadiamento da fibrose, podendo ser aplicados como métodos diagnósticos alternativos na HCC.
\end{abstract}

Introduction: Serum markers have been used in the assessment of liver fibrosis in patients with chronic hepatitis $\mathrm{C}(\mathrm{CHC})$. Aims: We evaluated the capacity of aspartate aminotransferase (AST)/alanine aminotransferase (ALT) ratio, gama-glutamyltransferase (GGT) levels, platelet count, the AST to platelet ratio index (APRI) and serum hyaluronic acid $(H A)$ to predict the intensity of hepatic fibrosis in patients with $C H C$ and the variation of these markers after therapy with interferon. Patients and methods: In 72 patients with hepatitis C, AST/ALT ratio, GGT levels, platelet count, the APRI index (calculated as the ratio of AST to platelets) and serum HA concentration were determined and compared to histological staging according to the scoring system of METAVIR. Sixty-five patients received interferon and ribavirin therapy. The individuals that completed the treatment $(\mathrm{n}=33)$ underwent a new test for serum marker of fibrosis in order to compare it with pretreatment levels. Results: GGT levels, platelet count, the APRI index and serum HA were correlated with the stage of hepatic fibrosis $(\mathrm{p}<0.01)$, except AST/ALT ratio. The analysis of the areas under the ROC curve $(A \cup C)$ evidenced that $A P R I$ and $H A$ levels were the markers with the best association with hepatic staging: $A \cup C(A P R I)=0.85$ and $A \cup C(H A)=0.86$. After therapy with interferon, only GGT and the APRI showed reduction of their levels $(\mathrm{p}<0.05)$. Conclusion: $H A$ and the APRI index were the most accurate approaches to liver fibrosis staging and they may be used as alternative diagnostic methods in patients with CHC.

key words

Serum markers

Hepatic fibrosis

Chronic hepatitis C

Hyaluronic acid

1. Mestranda em Clínica Médica da Universidade Federal de São Paulo (UNIFESP); médica hepatologista do Hospital Universitário da Universidade Federal de Alagoas (HU/UFAL).

2. Professor-doutor das disciplinas de Endocrinologia e Metabologia e de Biologia Molecular da UNIFESP.

3. Professora titular da Disciplina de Biologia Molecular da UNIFESP.

4. Professora-doutora adjunta da Disciplina de Castroenterologia da Fundação Universitária de Ciências da Saúde de Alagoas Covernador Lamenha Filho (UNCISAL).

5. Mestre em Anatomia Patológica; chefe do Serviço de Patologia do HU/UFAL.

6. Professora da Disciplina de Clínica Médica da UNIFESP; orientadora do Programa de Pós-graduação de Clínica Médica da Universidade Federal de São Paulo/Escola Paulista de Medicina (UNIFESPEPM).

Tese apresentada na UNIFESP para obtenção do título de Mestre em Clínica Médica, 2007. 


\section{Introdução}

A infecção pelo vírus da hepatite $\mathrm{C}(\mathrm{VHC})$ afeta cerca de 170 milhões de pessoas, aproximadamente $3 \%$ da população mundial( ${ }^{(9)}$. Estima-se que $54 \%$ a $86 \%$ dos pacientes infectados evoluam para a forma crônica, predispondo a complicações, como cirrose, falência hepática e hepatocarcinoma ${ }^{(23)}$.

A biópsia hepática é considerada o gold standard para o diagnóstico da fibrose na hepatite crônica $\mathrm{C}(\mathrm{HCC})^{(14)}$. Em geral, apesar de ser procedimento seguro, está sujeita a complicações, apresenta elevado custo e existem 10\% a $20 \%$ de discrepâncias entre os observadores sobre a avaliação da fibrose hepática. Essas dificuldades levaram ao desenvolvimento de métodos não-invasivos, reprodutíveis e de elevada acurácia na avaliação da gravidade da fibrose hepática ${ }^{(29)}$.

Alguns marcadores séricos de fibrose se destacam por apresentarem correlação com a histologia hepática e pela facilidade do seu uso clínico, como o índice aspartato aminotransferase (AST)/alanina aminotransferase (ALT), a gama-glutamiltransferase (GGT) e a contagem de pla-

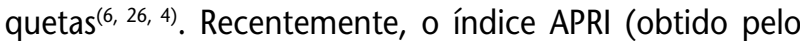
quociente AST/plaquetas) tem sido aplicado devido a sua elevada acurácia como preditor da fibrose hepática nos portadores de $\mathrm{HCC}(21)$. A medida do ácido hialurônico $(\mathrm{AH})$, apesar de não ser um teste bioquímico de rotina, tem se firmado como excelente marcador de fibrose hepática, por suas elevadas sensibilidade e especificidade ${ }^{(31,32)}$.

Os marcadores de fibrose têm sido utilizados também na monitoração terapêutica de pacientes com HCC. Esses pacientes têm apresentado regressão pós-tratamento do grau histológico de fibrose, atribuída à ação antifibrogênica do interferon alfa (IFN- $\alpha$ ), que atua na limitação da ativação das células estrelares e no aumento da produção de colagenases e metaloproteinases ${ }^{(2 ; 17)}$.

O objetivo deste estudo foi avaliar o valor preditivo desses marcadores séricos de fibrose hepática em portadores de HCC e sua variação pós-terapia antiviral.

\section{Material e métodos}

Realizou-se estudo longitudinal de caráter observacional em pacientes portadores de hepatite $C$ (ambos os sexos), com idades entre 18 e 75 anos, prospectivamente selecionados no Ambulatório de Hepatites Virais do Hospital Universitário da Universidade Federal de Alagoas (HU/UFAL), de junho a dezembro de 2005. Diagnosticou-se hepatite $C$ por meio de anticorpos anti-VHC/ensaio imunossorvente ligado à enzima (ELISA) de terceira geração (Abbott Laboratories, IMX ${ }^{\circledR}$ ), confirmado pela detecção do RNA/VHC por reação em cadeia da polimerase (PCR) (Amplicor, Roche Diagnostic Systems ${ }^{\circledR}$ ).

Foram excluídos pacientes com doença hepática descompensada, co-infectados com vírus da hepatite B (VHB) e da imunodeficiência humana (HIV), com relato de consumo de etanol > $40 \mathrm{~g} /$ dia. Para avaliação, utilizou-se anamnese, exame físico, provas de função hepática, ultra-sonografia do abdome superior e sorologias para hepatite viral B e HIV.

\section{Avaliação histológica}

Realizaram-se biópsias hepáticas percutâneas em todos os pacientes. Os espécimes foram corados por hematoxilina-eosina (HE); tricrômio de Masson, azul da Prússia (método de Perls) e impregnação das fibras reticulares pela prata (método de Gomori), sendo avaliados por um único patologista sem conhecimento prévio dos casos. A análise histológica baseou-se nos critérios METAVIR ${ }^{(1)}$, que consistem na análise do grau de fibrose (F0 a F4) e atividade necroinflamatória (A0-A3).

\section{Parâmetros hematológicos e bioquímicos}

Amostras sangüíneas foram coletadas com intervalo de até 72 horas antes da realização da biópsia hepática. Dosagens séricas de AST, ALT e GGT foram feitas por método cinético automatizado. Obteve-se a contagem de plaquetas por método automatizado em equipamento Cell-Dyn-Counter ${ }^{\circledR}$. Calculou-se o índice APRI pela fórmula: valores séricos de AST (/limite superior da normalidade) $x$ $100 /$ contagem de plaquetas (103/I).

Os níveis séricos do AH foram determinados pelo método ELISA-like, com base na afinidade do AH por proteínas específicas da cartilagem bovina desenvolvido no Laboratório de Biologia Molecular da Universidade Federal de São Paulo (UNIFESP), utilizando kits não comercializados. O valor de referência em estudo prévio avaliando 545 indivíduos normais variou de 1,1 a 97,1 ng/l. Em 40 pacientes portadores de cirrose hepática a variação foi de 96,5 a $4.580 \mathrm{ng} / \mathrm{l}$. A sensibilidade do método é bastante elevada, sendo capaz de detectar dosagens de $\mathrm{AH}$ de até $0,24 \mathrm{ng} / \mathrm{l} .{ }^{(11)}$

Sessenta e cinco pacientes foram tratados com IFN (61 receberam IFN peguilado alfa 2a, $180 \mathrm{mcg}, 1$ ampola 
subcutânea [SC] por semana, e quatro receberam IFN- $\alpha$, 3 milhões de unidades, 3 ampolas $\mathrm{SC} /$ semana) associado à ribavirina $250 \mathrm{mg}$ (4 comprimidos/dia); sete pacientes não apresentaram indicação terapêutica.

O tratamento foi mantido por 48 semanas e dosados os mesmos marcadores séricos de fibrose seis meses após a conclusão do tratamento antiviral.

\section{Análise estatística}

A correlação entre os dados semiquantitativos foi determinada usando o teste não-paramétrico de Spearman. Foram construídas curvas receiver operator characteristic (ROC) para avaliar o poder discriminativo de cada variável, sendo adotado nível de significância igual a 0,05 em todas as análises.

Para os pacientes que completaram o tratamento, utilizaram-se técnicas de análise de variância (ANOVA) com medidas repetidas do fator instante.

O estudo foi aprovado pelo Comitê de Ética da UFAL, endossado e reconhecido pela UNIFESP, porquanto os pacientes envolvidos na pesquisa procediam do ambulatório de hepatites virais do HU/UFAL. Todos os pacientes preencheram o termo de consentimento informado antes de serem incluídos no trabalho.

\section{Resultados}

As características gerais dos pacientes são apresentadas na Tabela 1. Para avaliar a capacidade diagnóstica dos marcadores estudados, agrupamos os pacientes de acordo com o estadiamento hepático (Tabela 2).

A análise do índice AST/ALT relacionado à fibrose tecidual não mostrou diferença significativa entre os grupos de pacientes com fibrose inicial ou avançada $(p>0,05)$.
Os demais marcadores séricos de fibrose (GGT, contagem de plaquetas, índice APRI e AH) apresentaram associação com o grau histológico de fibrose. Foram construídas curvas ROC e estimadas a sensibilidade, especificidade, valores preditivos positivo (VPP) e negativo (VPN) desses marcadores para diversos pontos de corte (Figuras 1 e 2; Tabela 3).

Verificou-se que o índice APRI e o AH apresentaram maior capacidade de discriminar os pacientes com grau avançado de fibrose, em relação aos demais marcadores.

Foram submetidos a tratamento antiviral com IFN e ribavirina 65 pacientes. Entre os que concluíram o tratamento $(n=33)$, avaliaram-se os marcadores AST/ALT, GGT, contagem de plaquetas, APRI e AH, sua relação com o grau de fibrose hepática inicial e a variação desses marcadores pré e pós-terapia antiviral (Tabela 4).

Não se detectou efeito de interação entre o instante de observação e o grau de fibrose em nenhuma das variáveis estudadas $(p>0,05)$. Ou seja, a diferença média entre os valores pré e pós-tratamento dos marcadores não dependeu do grau de fibrose.

Apresentaram resposta virológica sustentada (RVS) 19 pacientes $(57,6 \%)$ e 14 pacientes não responderam à terapia antiviral.

\begin{tabular}{lc} 
Tabela $\mathbf{1}$ & $\begin{array}{c}\text { Características demográficas e } \\
\text { histológicas dos pacientes }(n=\mathbf{7 2})\end{array}$ \\
\hline Parâmetro & Número $(n)$ \\
Idade (anos) & $53 \pm 9$ \\
Gênero (masculino/feminino) & $46 / 26$ \\
Tratados & 65 \\
Não-tratados & 7 \\
Grau de fibrose & \\
FO/F2 & $66,7 \%$ \\
F3/F4 & $33,3 \%$ \\
\hline
\end{tabular}

\section{Tabela 2 Marcadores séricos relacionados à fibrose tecidual $(n=72)$}

\begin{tabular}{lccc}
\hline \multicolumn{3}{c}{ Grau de fibrose } & \\
& F0/F2 & F3/F4 & 0,39 \\
AST/ALT & $0,97 \pm 0,64$ & $0,96 \pm 0,30$ & $<0,01$ \\
GGT $(U / I) *$ & $74,7 \pm 61,9$ & $135,2 \pm 83,2$ & $<0,01$ \\
Plaquetas $\left(x 10^{3} / \mathrm{mm}^{3}\right)^{*}$ & $218 \pm 64$ & $163 \pm 76$ & $<0,01$ \\
APRI* & $0,76 \pm 0,76$ & $2.34 \pm 2,20$ & $<0,01$ \\
AH $(\mathrm{ng} / \mathrm{ml})^{*}$ & $27,3 \pm 12,5$ & $68,9 \pm 41,2$ & \\
\hline
\end{tabular}

AST/ALT: índice aspartato aminotransferase/alanina aminotransferase; CGT: gama-glutamiltransferase; APRI: índice AST/plaquetas; AH: ácido hialurônico. ${ }^{*} p<0,05$. 


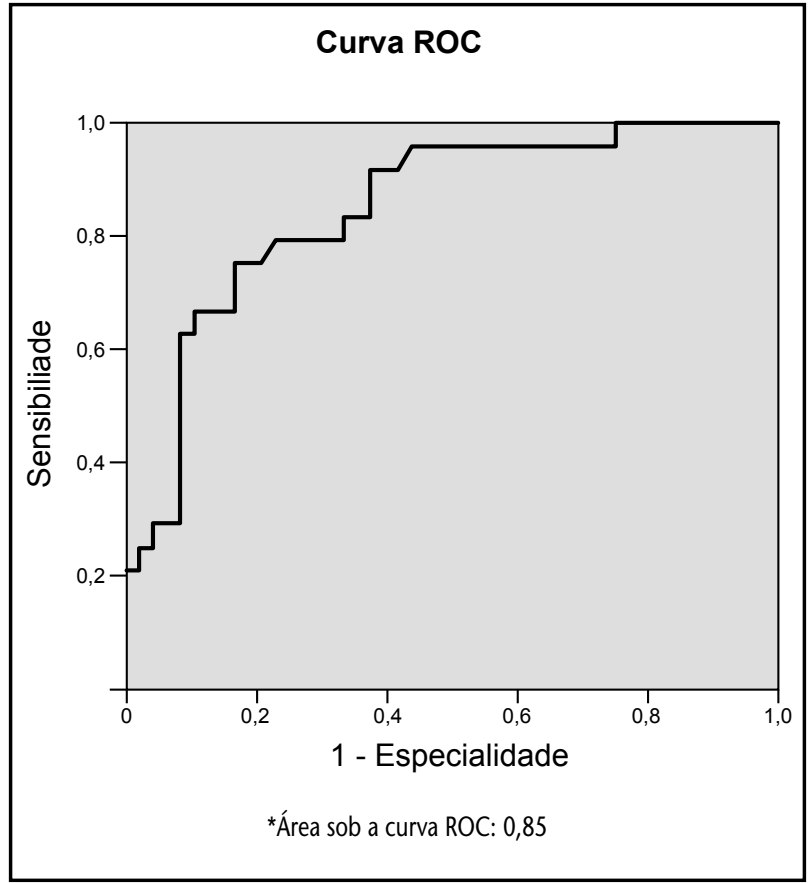

Figura 1 - Curva ROC desenvolvida para o parâmetro índice APRI

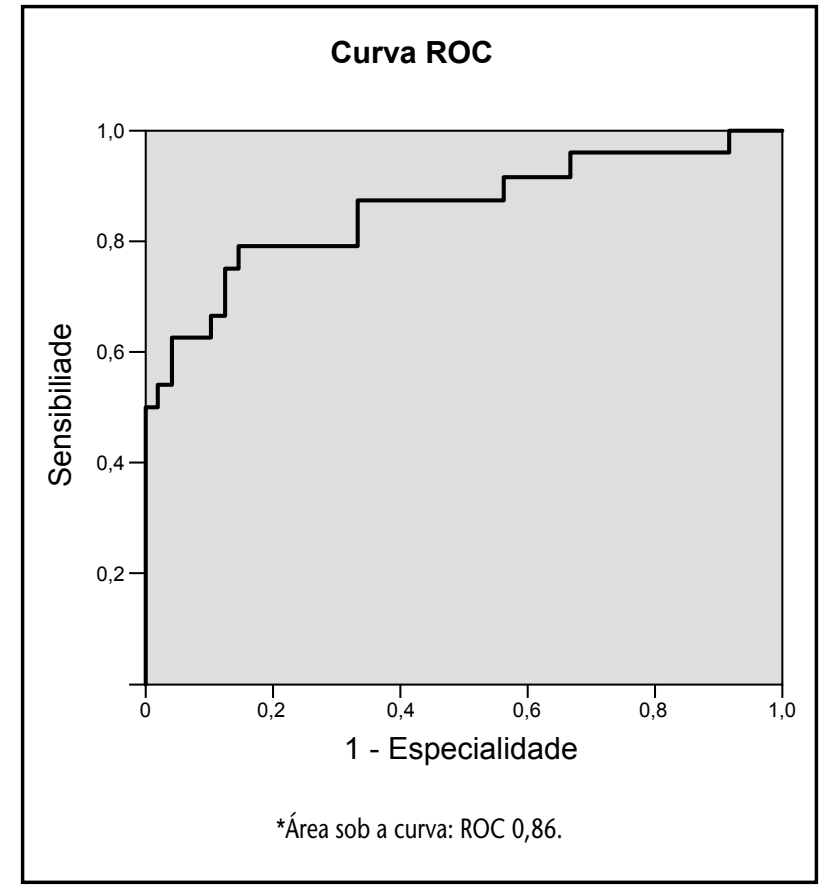

Figura 2 - Curva ROC desenvolvida para o parâmetro ácido hialurônico

\section{Área sob a curva ROC, sensibilidade, especificidade, valores preditivos positivo e negativo dos}

Tabela 3 marcadores estudados para diagnóstico de fibrose avançada ( $F \geq 2$ )

\begin{tabular}{lccccc}
\hline Marcador & AUC & Sensibilidade (\%) & Especificidade(\%) & VPP & VPN \\
\hline GGT $\geq 69 \mathrm{U} / /^{*}$ & 0,75 & 75 & 58,3 & 47 & 82 \\
Plaquetas $\leq 168 \times 103 / \mathrm{mm3}^{*}$ & 0,77 & 75 & 72,9 & 58 & 85 \\
APRI $\geq 0,84^{*}$ & 0,85 & 79,2 & 79,2 & 63 & 88 \\
$\mathrm{AH} \geq 40,1 \mathrm{ng} / \mathrm{ml}^{*}$ & 0,86 & 79,2 & 85,4 & 73 & 89 \\
\hline
\end{tabular}

AUC: Área sob a curva de concentração; GGT: gama-glutamiltransferase; VPP: valor preditivo positivo; VPN: valor preditivo negativo; APRI: índice AST/plaquetas; AH: ácido hialurônico.

${ }^{*} p<0,05$.

\section{Tabela 4 Média das variáveis em estudo, pré e pós-terapia antiviral $(n=33)$}

\begin{tabular}{|c|c|c|c|c|c|}
\hline \multirow[t]{2}{*}{ Variáveis } & \multirow[t]{2}{*}{ Grau de fibrose } & \multicolumn{4}{|c|}{ Instante de observação } \\
\hline & & Pré & DP & Pós & DP \\
\hline \multirow{2}{*}{ AST/ALT } & 0 a 2 & 1,1 & 0,7 & 1,7 & 1,4 \\
\hline & 3 ou 4 & 0,9 & 0,2 & 1,9 & 1,3 \\
\hline \multirow[t]{2}{*}{ GGT } & 0 a 2 & 67 & 68 & 50.8 & 49 \\
\hline & 3 ou 4 & 139 & 87 & 90 & 75 \\
\hline \multirow[t]{2}{*}{ Plaquetas $\left(x 10^{3}\right)$} & 0 a 2 & 219 & 64 & 195 & 64 \\
\hline & 3 ou 4 & 158 & 84 & 158 & 72 \\
\hline \multirow[t]{2}{*}{ APRI } & 0 a 2 & 0,7 & 0,6 & 0,4 & 0,3 \\
\hline & 3 ou 4 & 2,5 & 2,6 & 1 & 1,4 \\
\hline \multirow[t]{2}{*}{$\mathrm{AH}$} & 0 a 2 & 25 & 10,5 & 26 & 31 \\
\hline & 3 ou 4 & 63 & 39,5 & 47 & 32 \\
\hline
\end{tabular}

AST/ALT: índice aspartato aminotransferase/alanina aminotransferase; GCT: gama-glutamiltransferase; APRI: índice AST/plaquetas; AH: ácido hialurônico. DP: desvio padrão. 
Houve redução pós-tratamento dos níveis séricos de GGT e do índice APRI, com grau de significância estatística. O índice AST/ALT apresentou elevação pós-terapia antiviral. A contagem de plaquetas e AH sérico não apresentaram diferença significativa após uso do interferon.

\section{Discussão}

É necessária análise histológica de indivíduos com HCC, visando avaliações prognóstica e terapêutica desses pacientes. Sabe-se que aqueles que evidenciam estadiamento avançado podem progredir rapidamente para cirrose e hepatocarcinoma, enquanto pacientes sem fibrose ou apenas com fibrose portal apresentam progressão mais lenta, sendo desnecessária terapia antiviral na maioria dos $\operatorname{casos}^{(18)}$.

A biópsia hepática permanece como gold standard na avaliação da fibrose e da atividade histológica na $\mathrm{HCC}^{(5)}$. No entanto, constitui procedimento de custo elevado, com complicações ocasionais como dor, hemorragia e, em raros casos, morte ${ }^{(14)}$.

Os resultados dos estudos obtidos em diferentes populações confirmam que a aplicação de marcadores bioquímicos pode ter elevado valor preditivo no diagnóstico da fibrose significativa.

Neste estudo foram avaliados portadores de HCC, visando estabelecer a sensibilidade, especificidade e os VPPs e VPNs do índice AST/ALT, da GGT, da contagem de plaquetas, do índice APRI e do AH na determinação de graus avançados de fibrose hepática.

A análise de parâmetros histológicos dos 72 pacientes revelou que 48 deles apresentavam estádios iniciais da hepatopatia crônica, sendo a maior parte das biópsias hepáticas (40,3\%) classificadas como grau 2 de fibrose (F2), segundo os critérios de METAVIR. Um terço dos pacientes apresentava estádios mais avançados da doença hepática (F3 e F4). Desses, encontramos apenas cinco pacientes $(6,9 \%)$ na fase de cirrose hepática.

Para análise da associação entre os níveis séricos dos marcadores indiretos de fibrose e o estadiamento histológico, os pacientes foram divididos em grupos conforme 0 grau de fibrose.

Não se observou associação $(p=0,91)$ entre os valores do índice AST/ALT e os graus de estadiamento hepático. Assim, corroborando os nossos achados, Reedy et al. ${ }^{(20)}$ observaram, em 77 pacientes com HCC, que o índice AST/ALT $\geq 1$ foi encontrado em apenas dez pacientes dos 23 em fase de cirrose hepática.

Observamos que a média do nível sérico da GGT foi maior nos pacientes com grau de fibrose 3 ou $4(p<0,01)$. A melhor relação entre sensibilidade e especificidade ocorreu para o ponto de corte igual a $69 \mathrm{U} / \mathrm{l}$.

De modo semelhante, Koda et al.(9), ao avaliarem 360 portadores de HCC, obtiveram média da GGT de $69+66$ $\mathrm{U} / \mathrm{I}$ nos pacientes com fibrose F0 a F3. A elevação da GGT foi atribuída à presença de lesões em ductos biliares nos portadores de $\mathrm{HCC}^{(6)}$.

Em apenas 13 pacientes (18\%), encontramos contagem de plaquetas abaixo do limite inferior da normalidade $(<140$ $\left.\mathrm{mil} / \mathrm{mm}^{3}\right)$. Os valores normais foram atribuídos ao predomínio de pacientes num estádio inicial em nossa série. À semeIhança do que avaliamos, em outros estudos com prevalência de pacientes em estádios iniciais da hepatopatia, a contagem de plaquetas estava próxima da normalidade ${ }^{(3,24)}$. Entretanto, a trombocitopenia é uma complicação comum das hepatopatias crônicas em estádios avançados, tendo sido propostos, em sua patogênese, hipertensão portal, esplenomegalia, diminuição da produção de trombopoetina e elevação da imunoglobulina $\mathrm{G}(\lg G)$ associada a plaquetas ${ }^{(4)}$.

Nesta investigação verificou-se que 0 índice APRI foi maior nos pacientes com fibrose avançada (F3 e F4) $(p<0,001)$, sendo encontradas elevadas sensibilidade $\mathrm{e}$ especificidade para graus avançados de fibrose no ponto de corte igual a 0,84 .

Em estudo semelhante, Parise et al.(15) aplicaram o índice APRI na avaliação de pacientes com HCC e determinaram, pela curva ROC, que a sensibilidade e especificidade desse índice no diagnóstico de fibrose hepática avançada foram $85 \%$ e $66 \%$, respectivamente (APRI $\geq 0,70$ ). $A$ área sob a curva ROC (AUC) foi 0,82 , à semelhança do nosso resultado, cujo valor foi 0,85 .

Obtivemos níveis séricos de $\mathrm{AH}$ maiores nos pacientes com fibrose hepática avançada (F3 e F4). A análise dos resultados demonstrou que valores acima do ponto de corte determinado pela curva ROC $(40,1 \mathrm{ng} / \mathrm{ml})$ se associaram a graus mais avançados de fibrose hepática $(p<0,01)$, com sensibilidade de $79,2 \%$ e especificidade de $85,4 \%$, VPP de $73 \%$ e VPN de $89 \%$.

Vários estudos têm utilizado o AH como preditor de fibrose nos pacientes com $\operatorname{HCC}(7,12,13,15,27)$. Nossos achados corroboram os de Halfon et al. ${ }^{(7)}$, que reuniram 405 portadores de HCC para avaliar a acurácia desse marcador em predizer o estádio da fibrose hepática, e concluíram que o AH poderia ser usado clinicamente como marcador não-invasivo para fibrose e/ou cirrose.

$\mathrm{O}$ aumento das concentrações de $\mathrm{AH}$ nos pacientes com HCC pode refletir:

- estímulo da produção desse devido à inflamação e remodelação tecidual pela ativação dos lipócitos; 
- diminuição da depuração e degradação do AH, especialmente nas células endoteliais sinusoidais;

- progressão da fibrose com aumento pronunciado do tecido conjuntivo no trato portal dos portadores de hepatite crônica e cirrose hepática ${ }^{(8)}$.

Até a avaliação final deste estudo, 33 pacientes (do total de 65) haviam concluído o tratamento antiviral com IFN e ribavirina. Seis meses após o término do tratamento, os marcadores séricos foram novamente dosados e relacionados aos níveis pré-tratamento. Embora a avaliação da resposta terapêutica não fosse o objetivo deste estudo, foi realizado PCR/VHC seis meses após o término do tratamento antiviral. Assim, observamos RVS em 19 pacientes (57,6\%), entretanto 14 pacientes não responderam à terapia antiviral.

A biópsia hepática pós-tratamento não foi realizada por não fazer parte do protocolo de seguimento em curto prazo dos portadores de hepatite crônica viral C.

Obtivemos redução dos valores médios de GGT de $139,4 \pm 87,1 \mathrm{U} / \mathrm{l}$ para $89,9 \pm 75,2 \mathrm{U} / \mathrm{l}$ pós-tratamento em pacientes com estadiamento avançado. A variação da dosagem sérica da GGT com o tratamento antiviral tem sido avaliada de forma isolada ou associada a outros índices de fibrose ${ }^{(19)}$. Redução em seus níveis é atribuída à melhora da colestase hepática, contudo os valores da GGT diferem significativamente entre pacientes respondedores e nãorespondedores ao interferon ${ }^{(19 ; 24)}$.

Verificamos redução do índice APRI pós-tratamento, tanto nos pacientes com fibrose inicial quanto no estádio avançado. À semelhança do que observaram Yu et al.(33), avaliando 776 pacientes com HCC submetidos ao tratamento com interferon.
Em nosso estudo, os níveis séricos de GGT e do índice APRI apresentaram redução pós-terapia antiviral, com grau de significância estatística. Esse achado sugere reversibilidade da fibrose hepática por ação do IFN, que somente poderia ser confirmada mediante reavaliação histológica hepática pós-tratamento.

Os níveis médios do AH foram maiores nos indivíduos com estadiamento avançado, mas, apesar de ter havido redução do $\mathrm{AH}$ pós-tratamento, essa não foi significante.

Resultado semelhante foi obtido por Poynard et al. ${ }^{(19)}$ na avaliação de 165 portadores crônicos do VHC submetidos à terapia com IFN, não havendo variação estatisticamente significativa entre a dosagem inicial e os valores pós-tratamento. Entretanto, alguns autores encontraram redução dos valores do AH mais evidente nos pacientes que apresentaram resposta virológica sustentada e naqueles com reversão da fibrose ${ }^{(25,29)}$.

Para finalizar, em nosso trabalho encontramos relação entre os marcadores séricos de fibrose: AH, GGT, contagem de plaquetas e índice APRI com o estadiamento histológico (padrão-ouro). Nossos resultados permitem inferir que os marcadores séricos estudados, especialmente o $\mathrm{AH}$ e o índice APRI, sugerem o estadiamento hepático nos pacientes com contra-indicação à realização de procedimentos invasivos e, eventualmente, contribuem para a monitoração da terapia antiviral.

Outros estudos validando os marcadores séricos de fibrose hepática são necessários para que esses sejam incorporados ao diagnóstico e seguimento dos pacientes portadores de hepatopatia crônica de forma padronizada.

\section{Referências}

1. BEDOSSA, P. Presentation of a grid for computer analysis for complilation of histopathologic lesions in chronic vira hepatitis C. Ann Pathol, v. 13, n. 4, p. 260-5, 1993.

2. BUENO, M. R.; DANEREI, A.; ARMENDARIZ-BORUNDA, J. Cholestasis-induced fibrosis is reduced by interferon alpha-2a and is associated with elevated liver metalloprotease activity. J Hepatol, v. 33, n. 6, p. 915-25, 2000

3. ESMAT, G. et al. Evaluation of serum biomarkers of fibrosis and injury in Egyptian patients with chronic hepatitis C. J Hepatol, v. 46, p. 620-7, 2007.

4. FUSEGAWA, H. et al. Platelet activation in patients with chronic hepatitis C. Tokai J Exp Clin Med, v. 7, n. 4, p. 101-6, 2002.
5. GEBO, K. A. et al. Role of liver biopsy in management of chronic hepatitis C: a systematic review. Hepatology, v. 36, n. 5, suppl. 1, p. S161-72, 2002.

6. GIANNINI, E.; RISSO, D; TESTA, R. Transportability and reproducibility of the AST/ALT ratio in chronic hepatitis C patients. Am J Gastroenterol, v. 96, p. 918-9, 2001.

7. HALFON, P. et al. Accuracy of hyaluronic acid level for predicting liver fibrosis stages in patients with hepatitis C virus. Comparative Hepatology, v. 4, p. 6, 2005.

8. ICHIDA, T. et al. Localization of hyaluronan in human liver sinusoids: a histochemical study using hyaluronanbinding protein. Liver, v. 16, p. 365-71, 1996.

9. KODA, M. et al. Fibrolndex, a practical index for predicting 
significant fibrosis in patients with chronic hepatitis $C$. Hepatology, v. 45, n. 2, p. 297-306, 2007.

10. LAUER, G.M.; WALKER, B. D. Hepatitis C viral infection. N Engl J Med, v. 345, n. 1, p. 41-52, 2001.

11. MARTINS, J.R. et al. Practical determination of hyaluronan by a new noncompetitive fluorescence-based assay on serum of normal and cirrhotic patients. Anal Biochem, v. 319, n. 1, p. 65-72, 2003.

12. McHUTCHISON, J.G. et al. Measurement of serum hyaluronic acid in patients with chronic hepatitis $\mathrm{C}$ and its relationship to liver histology. J Gastroenterol Hepatol, v. 15, n. 8, p. 945-51, 2000.

13. MURAWAKI, Y. et al. Diagnostic value of serum type IV collagen test in comparison with platelet count for predicting the fibrotic stage in patients with chronic hepatitis. J Gastroenterol Hepatol, v. 16, p. 777-81, 2001.

14. OLGA, O.; NIKOLAI, D. Invasive and non-invasive monitoring of hepatitis $\mathrm{C}$ virus induced liver fibrosis: alternatives or complements? Curr Pharm Biotechnol, v. 4, 195-209, 2003.

15. PARISE, E. R. et al. Noninvasive serum markers in the diagnosis of structural liver damage in chronic hepatitis C vírus infection. Liver Int, v. 26, n. 9, p. 1095-9.

16. POHL, A. et al. Serum aminotransferase levels and platelet counts as predictors of degree of fibrosis in chronic hepatitis C virus infection. Am J Gastroenterol, v. 96, n. 11, p. 3142-6, 2001.

17. POL, S. et al. Reversibility of hepatitis $C$ virus related cirrhosis. Hum Pathol, v. 35, n. 1, p. 107-12, 2004.

18. POYNARD, T. et al. Fibrosis in patients with chronic hepatitis C: detection and significance. Sem Liver Dis, v. 20, p.47-55, 2000

19. POYNARD, T. et al. Biochemical markers of liver fibrosis in patients infected by hepatitis $C$ virus: longitudinal validation in a randomized trial. J Viral Hepat, v. 9, n. 2, p. 128-33, 2002

20. REEDY, D. W.; LOO, A.T.; LEVINE, R. A. AST/ALT ratio > or $=1$ is not diagnostic of cirrhosis in patients with chronic hepatitis C. Dig Dis Sci, v. 43, n. 9, p. 2156-9, 1998.

21. RYDER, S. D. et al. Progression of hepatic fibrosis in patients with hepatitis C: a prospective repeat liver biopsy study. Gut, v. 53, n. 3, p. 451-5, 2004.
22. SCHIAVON, L. L. et al. Simple blood tests as noninvasive markers of liver fibrosis in hemodialysis patients with chronic hepatitis $C$ virus infection. Hepatology, v. 46,n. 6, p. 307-14, 2007.

23. SEEF, L. Natural history of hepatitis C. Hepatology, v. 36, p. 35-46, 2002.

24. SERFATY, L. et al. Factors predictive of the response to interferon in patients with chronic hepatitis C. J Hepatol, v. 21, p. 12-7, 2004.

25. SERPAGGI, J. et al. Direct and indirect evidence for the reversibility of cirrhosis. Hum Pathol, v. 37, n. 12, p. 1519-26, 2006.

26. SILVA, I. S. et al. Role of gamma-glutamyl transferase activity in patients with chronic hepatitis $\mathrm{C}$ vírus infection. J Gastroenterol Hepatol, v. 19, n. 3, p. 314-8, 2004.

27. TAKAMATSU, S. et al. Noninvasive determination of liver collagen content in chronic hepatitis: multivariate regression modeling wuth blood chemical parameters as variables. J Gastroenterol, v. 32, n. 3, p. 355-60, 1997.

28. TANIGUCHI, H. et al. Long-term monitoring of platelet count, as a non-invasive marker of hepatic fibrosis progression and/or regression in patients with chronic hepatitis C after interferon therapy. J Gastroenterol Hepatol, v. 21, n. 1, p. 281-7, 2006.

29. UENO, T. et al. Long-term follow-up of interferon-treated chronic hepatitis $\mathrm{C}$ and serum hepatic fibrosis markers. Hepatogastroenterology, v. 48, n. 40, p. 1124-8, 2001.

30. WAI, C. T et al. A simple noninvasive index can predict both significant fibrosis and cirrhosis in patients with chronic hepatitis C. Hepatology, v. 38, n. 2, p. 512-26, 2003.

31. WONG, V. S. et al. Serum hyaluronic acid is a useful marker of liver fibrosis in chronic hepatitis $C$ virus infection. $J$ Viral Hepatitis, v. 5, n. 3, p. 187-92, 1998.

32. YAMADA, M. et al. Serum hyaluronan as a marker of liver fibrosis in hemophiliacs with hepatitis $\mathrm{C}$ virus associated chronic liver disease. Acta Haematol, v. 99, n. 4, p. 212-6, 1998.

33. YU, M. L. et al. Simple noninvasive index for predicting long-term outcome of chronic hepatitis C after interferon-based therapy. Hepatology, v. 44, n. 5, p. 1086-97, 2006. 